\title{
Padres y madres adolescentes en el ejercicio de la crianza
}

\author{
Adolescent fathers and mothers in the parenting exercise
}

Pais adolescentes no exercício da parentalidade

\author{
Doris Amparo Parada-Rico* \\ Carlos Iván García-Suárez**
}

Para citar este artículo / To reference this article / Para citar este artigo/

Parada-Rico DA, García-Suárez CI. Adolescent fathers and mothers in the parenting exercise. Rev. cienc. cuidad. 2017; 14(2): 113-129.

\section{Resumen}

Introducción: En general, se ha considerado la crianza como acciones de socialización dirigidas por adultos, estimando a los adolescentes como personas no aptas para lograr las trayectorias de desarrollo ideal esperado en los niños y niñas; de otra parte, el Estado, a pesar de avanzar por la equidad para estas personas, en ocasiones invisibiliza sus derechos y necesidades. Materiales y Métodos: A través de la revisión sistemática de documentos y bases de datos como ScienceDirect, Scopus, Dialnet, Pubmed, Proquest, adolec; se indagó información en español, inglés y portugués de los últimos diez años con las palabras clave: prácticas de crianza y adolescentes, padres-madres adolescentes y políticas públicas en adolescencia; esta revisión retomó 84 publicaciones con los aspectos señalados. Resultados: Se identifican percepciones de los y las adolescentes como padres y madres, sus interacciones sociales en el ejercicio de la crianza, pautas y prácticas de crianza y los aportes que frente a su reconocimiento como adolescentes padres establece el Estado. Conclusiones: Es necesario identificar las percepciones en adolescentes padres y madres y construir conjuntamente con éstos Políticas Públicas que conlleven al aumento de redes de apoyo para asumir las nuevas tareas del cuidado y continuar con las actividades que le imponen los modelos y sistemas sociales.

Palabras clave: Adolescente, crianza del niño, derechos reproductivos, relaciones padres-hijo (Fuente: DecsBireme)

\section{Abstract}

Introduction: In general, parenting has been considered as the actions of socialization led by adults, which consider teenagers as unable people to achieve trajectories of the expected ideal development for girls and boys; on the other side the State despite of making progress about equity of these people, often turns their rights and necessities invisible. Materials and Methods: Through a systematic review of documents and databases such as ScienceDirect, Scopus, Dialnet, Pubmed, Proquest, Adolec; information in Spanish, English and Portuguese of the last ten years was gathered with keywords: parenting practices and teenagers, teenage mothers-fathers, public policies in adolescence; this review returned 84 publications with the pointed aspects. Results: Perceptions of the adolescent mothers and fathers are identified, their social interactions in the parenting exercise, guidelines and practices of parenting and the contributions that regarding their recognition as adolescent parents, the State establishes. Conclusions: It is necessary to identify the perceptions in both adolescent fathers and mothers, and build jointly Public Politics that lead to the increase of support networks to assume the new tasks of care and continue with the activities that the models and social systems impose.

Keywords: Adolescent, parenting, reproductive rights, parent-child relationship (Source: DecsBireme) 
ISSN-PRINT

1794-9831

E-ISSN 2322-7028

Vol. 14 No. 2

Jul - Dic 2017

Cúcuta, Colombia

\section{Resumo}

Introdução: De maneira geral tem-se considerado a parentalidade como ações de socialização dirigidas por adultos, estimando aos adolescentes como pessoas não aptas para conseguir as trajetórias de desenvolvimento ideal que esperamos nas crianças; de outra parte o Estado apesar de avançar pela equidade para estas pessoas, em ocasiões faz invisível seus direitos e necessidades. Materiais e Métodos: Através de revisão sistemática de documentos e bases de dados como ScienceDirect, Scopus, Dialnet, Pubmed, Proquest e Adolec; indagou-se informação em espanhol, inglês e português dos últimos dez anos com as palavras chave: práticas parentais e adolescentes, pais adolescentes, políticas públicas em adolescência; esta revisão retomou 84 publicações com os aspectos identificados. Resultados: Identificaram-se percepções dos adolescentes como pais, suas interações sociais no exercício da parentalidade, pautas e práticas parentais e as contribuições que estabelece o Estado para seu reconhecimento como pais adolescentes. Conclusões: É necessário identificar as percepções dos pais adolescentes, e construir conjuntamente com estas Políticas Públicas, para que levem ao aumento de redes de apoio para assumir as novas tarefas do cuidado e continuar com as atividades que lhe impõem os modelos e sistemas sociais.

Palavras-chave: Adolescente, direitos reprodutivos, parentalidade, relações pai-filho (Fonte: DecsBireme)

\section{Introducción}

Inicialmente, para realizar el presente análisis frente al tema de la crianza en la adolescencia y de las apuestas que hacen las normativas nacionales e internacionales en este aspecto, debe tenerse en cuenta la definición de adolescencia que, según el informe "The State of the World's Children 2011" (1), resulta difícil enunciar por diversas razones.

Entre ellas porque, para algunos autores y disciplinas, esta es una transición en el curso de la vida que debe ser vista desde una perspectiva más constructivista ${ }^{1}$. Entre los autores se cuenta a Duarte (3), Varela y Lara (4), Climent (5) e Higuita et al. (6), los cuales la identifican como la construcción de una urdimbre que se gesta de acuerdo con el contexto social, político, histórico y cultural y no como un periodo fijo en el cual termina la niñez y se inicia la adolescencia.

De otra parte, y desde visiones más positivistas ${ }^{2}$, se han hecho definiciones al respecto que se originan en el marco jurídico y de salud y, aunque no confluyen de manera exacta en su delimitación, entienden la adolescencia como una etapa etaria prefijada y homogénea que se relaciona con los alcances de la

1 The constructivist perspectives emphasizes in the premises of a nonradical conception, in which reality appears as a human construction that informs about the relations between individuals and their context. The individual appears as a social product -Homo Socius-(2).

2 The positivist vision: is characterized for being rational, factual, based on the observable, in the manipulable and verifiable (7) denominada mayoría de edad y con transiciones físicas y biopsicosociales que deben recorrerse para lograr la juventud o adultez.

En Colombia, por ejemplo, el artículo 3 de la Ley 1098 de 2006 (8), Código de Infancia y Adolescencia, la demarca entre los 12 y los 18 años de edad. Sin embargo, se encuentran definiciones desde otras normas que se cruzan entre sí, como por ejemplo la Ley Estatutaria de Juventud (Ley 1622 de 2013) (9), que hace referencia a la definición de joven como la persona entre 14 y 28 años cumplidos, lo cual abarca una franja más amplia de edad en el rango clasificado como adolescencia.

En el ámbito de la salud, la Organización Mundial de la Salud (OMS), definió en 1995 la adolescencia como una época que se prolonga entre los 10 y los 19 años de edad, clasificada a su vez en categorías como adolescencia temprana (10-13 años), media (14-16 años) y tardía (17-19 años) (1-10).

En ese sentido, es difícil definirla de manera hegemónica, por lo cual es necesario reconocer que el ser humano durante el tránsito del curso de su vida posee características particulares como edad, sexo y raza, entre otras, pero además interacciona en un contexto, en general, que permea sus particularidades y que conlleva a unos modos de ser y a unas prácticas y saberes diversos, los cuales, como lo expresan Berger y Luckman (11), se van legitimando dentro del universo simbólico y van posibilitando el 
ordenamiento de las diferentes fases de la biografía: la niñez, la adolescencia, la adultez, etc.

Por lo anterior, se definen también en las comunidades o grupos humanos unas prácticas, modos de actuar o un deber ser, de acuerdo con parámetros establecidos en cada lugar, historia y contexto cultural. Esto permite entender, en relación con la crianza, cómo se pueden ir construyendo prácticas de manera diversa, según contextos específicos y tránsito en el curso de vida de las personas que la ejercen.

De otra parte, teniendo en cuenta la normatividad vigente y los alcances que ha tenido el Estado y las organizaciones internacionales como reguladores de la población, debe hacerse referencia a algunas bases legales que aprueban las uniones y el tener hijos e hijas durante este trayecto del curso de vida, así como las oportunidades y posibilidades que se presentan para estas personas.

A nivel internacional, la Convención Iberoamericana de Derechos de los Jóvenes (clasificados etariamente entre los 15 y 24 años por esta norma), adoptada por Colombia a partir del 1 de marzo de 2008, hace referencia en su artículo 20 a que éstos tienen derecho a la "formación de una familia, a la libre elección de la pareja, a la vida en común y a la constitución del matrimonio dentro de un marco de igualdad de sus miembros, así como a la maternidad y paternidad responsables..." (12).

Así mismo, en Colombia, en el literal 2 del artículo 140 del Código Civil (13), (actualizado según Sentencia C-507-04 del 25 de mayo de 2004), se valida el matrimonio de personas a partir de los 14 años -edad clasificada dentro del rango adolescente-, siempre y cuando para ello medie el permiso de sus padres.

De esta manera, se legitima jurídicamente que los adolescentes pueden casarse, unirse, tener hijos e hijas. No obstante, existen algunas apreciaciones en el contexto que devienen en estigma hacia ellos, en razón a si se unen, se encuentran en gestación o ejercen la crianza, pues en algunos casos se les asume como irresponsables y se considera que, por no haber llegado a la etapa adulta, no son aptos o eficaces para asumir este rol (14-18).

En cuanto a la edad y al género, se observa una tendencia a responsabilizar a la mujer de procesos como la natalidad, la anticoncepción y la crianza, haciendo énfasis, además, en el riesgo que significa ser mujer adolescente gestante o con hijos (19).

En Colombia, con respecto a la anticoncepción, ésta aparece más enfáticamente como consecuencia de varios acontecimientos: uno de ellos es la creación de Profamilia, en la década del 60, que inicia la oferta de servicios en los que la mujer es partícipe más activa en relación con el hombre de la utilización de este tipo de controles para disminuir la natalidad (20).

Además de lo anterior, se realiza en esta misma década, en Colombia, el censo de 1964, el cual evidenció que las colombianas tenían un promedio de siete hijos (21), siendo la población entre 10 y 19 años de casi 4 millones ( $23 \%$ de la población total), la cual se incrementó, siendo para el año 2000 de 7.8 millones, es decir, uno de cada cinco colombianos era adolescente (21).

En ese sentido, había que buscar estrategias para controlar su aparición y reproducción, pues en este tránsito del curso de vida estaba definida científicamente la aparición de los caracteres sexuales, lo que hacía concebir a la adolescencia como "susceptible de conductas desviadas y otras patologías relacionadas con la sexualidad" como lo refiere Ariza (22).

De otra parte, históricamente y en relación con las responsabilidades que han sido asignadas a la mujer, se ha establecido desde una visión patriarcal la división de trabajos, en la cual se identifica a ésta como la esposa y madre, ángel del hogar, eje de la familia y guardiana de las buenas costumbres (23), designaciones que, en definitiva, van inculcando en la mujer la labor del cuidado de sus hijos y, de otro lado, desvinculan al padre de estas acciones.

La definición de madre cuidadora de sus hijos e hijas es concebida de esta manera como un paradigma que establece para la mujer el cuidado del hogar y de los hijos, invisibilizando en algunos casos al hombre en las labores de la crianza y en las interacciones que se requieren en este contexto $\mathrm{y}$, más enfáticamente, al adolescente padre, pues se considera que es la mujer quien sabe realizar estas tareas, mientras que el hombre debe trabajar o continuar sus estudios para mejorar los ingresos, pues es su obligación.
E-ISSN 2322-7028

Vol. 14 No. 2

Jul - Dic 2017

Cúcuta, Colombia 
ISSN-PRINT

1794-9831

E-ISSN 2322-7028

Vol. 14 No. 2

Jul - Dic 2017

Cúcuta, Colombia
Lo anterior, según Zicavo y Fuentelba (24), puede tener consecuencias "más allá de la concepción social que entiende al padre como prescindible y secundario en la crianza, con el riesgo de producir padres ausentes y desentendidos de su función" y termina por legitimar la ausencia paterna. De otra parte, según Vega (25), se hacen invisibles sus voces, impidiendo conocer percepciones o necesidades presentes que apunten de manera real al desarrollo de programas o políticas públicas y que aporten a esta población (25).

Otro aspecto a resaltar es la insistente marcación de indicadores de riesgo que demuestran a la adolescencia en gestación como problema de salud pública, tal como lo refiere el Conpes Social 147 de 2012 (26), que expresa "la ocurrencia de embarazos a temprana edad ha sido considerada, desde la década de los 70, una problemática social y de salud pública necesaria de ser intervenida".

No obstante, cabe resaltar que, al analizar diferencias entre mujeres adultas y adolescentes en el proceso de la gestación, se ha demostrado que no hay contundencia ni diferencias significativas frente a la mortalidad materna en todos los rangos de edad (2729).

Althabe et al. (27), por ejemplo, identificó que el embarazo en las adolescentes no se asocia con un mayor riesgo materno, sino con mal pronóstico neonatal, pero sobre todo en las adolescentes más jóvenes ( $<15$ años), debiendo tenerse en cuenta el contexto geográfico en el que habitan.

También aparecen estudios que demuestran la importancia de realizar evaluación tanto de los factores de riesgo obstétrico como de los factores de riesgo psicosociales críticos determinantes (ansiedad severa y ausencia de apoyo social familiar), pues realmente se incrementa significativamente la probabilidad de identificar a las mujeres embarazadas con mayor riesgo de presentar complicaciones maternas y perinatales, como lo establecen Herrera et al (30), y no es la edad como variable única la que marca pautas de riesgo.

Lo anterior permite abrir un espacio para analizar si se ha estado señalando a todo el grupo de adolescentes como de riesgo, cuando en realidad son las condiciones particulares y sus interacciones sociales las que pueden desencadenar estos resultados adversos. Por lo tanto, se debe examinar cada situación contextualmente, evitando determinaciones homogeneizantes, pues, como lo refieren Miller y Arvisu (31), debe someterse a discusión el curso de vida normativo como un modelo vigente para todo contexto y toda temporalidad.

Con respecto a la crianza, se considera que las madres, padres y otros cuidadores como los hijos mayores aprenden a interactuar con el niño de acuerdo con cada ámbito cultural y, en ese sentido, como lo refiere Giddens (32), los cuidadores responden a las determinaciones socioculturales, las cuales son tenidas en cuenta como marco de referencia para organizar acciones en relación con el contexto que lo demanda, así como para garantizar la supervivencia de los niños y facilitar su bienestar (33-34).

Por lo anterior, la presente revisión documental permitirá la construcción del estado del arte de la crianza ejercida por padres y madres adolescentes y su relación frente a las oportunidades que le brinda el Estado para ejercerla. Se identifican las autopercepciones que hacen de sí los adolescentes padres y madres, así como sus interacciones con el contexto educativo, económico, de salud, familiar y de recreación con el cual se relacionan ejerciendo esta labor.

Este avance documental sirve de base para el análisis de la construcción de políticas públicas, normativas y programas ofertados para las personas en este trayecto del curso de vida y para el diseño de estrategias de reconocimiento en tanto adolescentes padres y madres. Es decir, el interpretar, comprender y reconocer al otro con sus diferencias $-\mathrm{y}$ a las generaciones con sus rasgos distintivos-, permitirá el diseño de políticas pertinentes e incluyentes, de acuerdo con las necesidades particulares del sujeto y el contexto.

\section{Metodología}

Para realizar el presente documento de revisión descriptiva se indagó información publicada en el año 2016 en revistas indexadas nacionales e internacionales, en los últimos 10 años (2006-2016), así como documentos y normas que aportaran al tema central de estudio. Se utilizó para ello el buscador google académico y bases de datos como Scielo, ScienceDirect, Scopus, Dialnet, Pubmed, Proquest y 
Adolec, con los siguientes descriptores: prácticas de crianza y adolescentes, padres-madres adolescentes, políticas públicas en adolescencia, parenting, parentalidade pais adolescentes. Esta búsqueda permitió el hallazgo de 105 publicaciones con los aspectos señalados, en idiomas español, inglés y portugués.

Posteriormente, se diseñó una matriz en Excel para clasificar la información, teniendo en cuenta, además, el país, año de publicación, metodología de investigación utilizada, categorías de abordaje y hallazgos principales. Igualmente, se abordaron autores clásicos en el tema. Esta categorización hizo posible filtrar la información, seleccionando aquellos documentos que atendieran y fueran pertinentes con la temática, verificando la validez de sus resultados y que, por actualización de contenidos, se encontraran publicados en los últimos diez años.

De esta manera, se consolidaron los artículos y publicaciones de los últimos diez años, obteniendo un total de 84 , los cuales fueron realizados en países como Colombia, Venezuela, Bolivia, Brasil, Chile, Argentina, Uruguay, México, Cuba, España y Estados Unidos.

\section{Percepción de sí como padres y madres adolescentes}

La construcción de la percepción de sí como padres o madres adolescentes en el ejercicio de la crianza se produce de acuerdo con contextos históricos, culturales y sociales, con marcas propias y significados para cada generación. Aún desde la gestación, en la cual se inician en la crianza, estas personas perciben cambios en sus planes de vida y, de esta manera, se configuran como madres o padres desde sus saberes en masculinidades y feminidades, como lo refiere Ortega (35).

De otra parte, en algunas poblaciones rurales, aunque no puede determinarse de manera homogénea, la construcción social de las identidades masculinas y femeninas como padres y madres se proyecta y está marcada, desde la infancia, por patrones sexistas tradicionales y estereotipados (36). Según Miguélez (37), mientras los padres invierten más tiempo en actividades recreativas o académicas, las madres se encargan de los cuidados primarios básicos como higiene y alimentación durante los primeros meses de su hijo(a), lo cual está marcado como un patrón que, desde su percepción como mujer u hombre, hace cada uno de sí.

En ese sentido, las mujeres van construyendo diversas percepciones de sí mismas como madres ejerciendo la crianza, como lo refieren Coronado y Ortiz (38), González y Estupiñan (39), Rozo et al. (40) y Andrade et al. (41), quienes destacan cómo en algunos casos estas mujeres retoman de sus madres concepciones y formas para asumir la crianza y, sin embargo, resignifican y modifican los vínculos con sus hijos percibiéndose como autónomas para realizar estas labores y tomar decisiones en el cuidado de ellos, se muestran felices y afables aunque, en ciertos casos, requieren del apoyo de sus madres o familia para llevar a cabo acciones en torno a este cuidado.

Para otras mujeres, el ser madre no influye de modo directo en su manera de percibirse y valorarse a sí mismas en relación con las adolescentes no madres, como lo establecen Reyes et al. (42), razón por la cual continúan llevando a cabo las acciones que realizaban antes de tener hijos, en el contexto que les rodea.

De esta forma, la crianza durante su adolescencia se convierte en una práctica concebida como normal, que las hace sentirse realizadas y con un nuevo estatus social que, según Serrano y Sánchez (43), les implica una mayor responsabilidad y les infunde deseos de progresar, pues las "responsabilidades maternas las hacen más madres..." (43), terminando incluso en el distanciamiento de sus amigos y dejando de lado actividades sociales para asumir responsabilidades de la crianza, como también lo identifican Gómez et al. (44) y Akiko (45).

De otra parte, esta práctica les facilita adquirir una nueva posición frente a su grupo de pertenencia y es un medio para tener algo propio, puesto que tener y criar un hijo o hija eleva la autoestima de la mujer, le demuestra haber superado la niñez y le otorga la posibilidad del ejercicio legal de la sexualidad (35, 41, 44). Akiko (45), incluso identifica que en ellas desaparecen sentimientos de vacío que existían con relación a su vida, aludiendo a que ahora tienen una razón para vivir.

Sin embargo, Reyes et al. (42), con respecto a la mujer adolescente que se encuentra gestando y en el ejercicio del cuidado de sus hijos, refiere la clasificación que determina distintas actitudes asumidas por ellas desde 
ISSN-PRINT

1794-9831

E-ISSN 2322-7028

Vol. 14 No. 2

Jul - Dic 2017

Cúcuta, Colombia la gestación, según su grupo etario. Por ejemplo, en la "adolescencia temprana (de 10 a 13 años), percibe como una fantasía este proceso, no proyecta planes a futuro con su pareja y puede llegar a mostrar trastornos emocionales: depresión y aislamiento" (42).

"En tanto que, en la adolescencia media (de 14 a 16 años), la actitud puede ser ambivalente, de orgullo y de culpa; asimismo, puede percibirse como una amenaza o una oportunidad que le brindará madurez" (42) y pueden "ver al padre como una esperanza para el futuro. Si el embarazo ocurre en la adolescencia tardía (de 17 a 19 años), la maternidad es apreciada como una forma de consolidar la intimidad con la pareja y comprometer al padre como un futuro compañero; además, las jóvenes desarrollan una gran preocupación por su papel de madres" (42).

En este sentido, "los patrones de comportamiento de las madres adolescentes hacia sus hijos pueden ir modificándose con el paso del tiempo y por la interacción que entablan con otros adultos implicados en el cuidado" (42).

Para los hombres adolescentes, la crianza es una experiencia nueva que es aprendida y socializada (46). Como perspectiva, la crianza visibiliza y resignifica el mundo del joven, incluyendo su concepción de sí mismo, sus relaciones sociales y le entrega un conjunto de prácticas de padre (47).

Arzola y Lombardo (48) refieren que existe actualmente una emergencia en la apropiación como padres “... Y es que ahora los hombres adolescentes se están convirtiendo en padres, cosa que no aparecía en tiempos anteriores, ellos no tenían hijos, las que sí los tenían eran las mujeres adolescentes".

Esta aseveración hace pensar en el marcado paradigma patriarcal y sexista que puede o que pudo aparecer en algunos lugares, en el que la mujer solamente es la encargada de cuidar y criar hijos e hijas, pues no existe la posibilidad de ver al hombre en labores de crianza (48). Sin embargo, actualmente emergen nuevas formas familiares y nuevas percepciones como padres.

Dentro de la información hallada, se identifican diversas percepciones de padres adolescentes frente al ejercicio de la crianza, en las cuales se pone de manifiesto que, para ellos, el asumir la crianza de hijos e hijas puede conllevar sentimientos de ambivalencia, puesto que hacen referencia a la idea de "responsabilidad y ruptura" (46).

La responsabilidad, como construcción social, tiene que ver con el desempeño de actividades de un buen padre que, imaginariamente, se relacionan con la protección económica, el cuidado, la atención de los hijos y de la pareja y que, a su vez, le hacen sentir que posee "la hombría adulta" $(20,35,45,46,47)$.

La ruptura hace referencia a una escisión de su adolescencia y se contrapone al ideal adolescente de libertad, conquista y competencia, donde la paternidad le permite al varón transitar y reconocerse simbólicamente como adulto pero le impide continuar sus actividades consideradas propias del adolescente $(35,42,45,46,47,49,50)$.

Ser padre adolescente y ejercer la crianza también puede percibirse como haber logrado la completitud de sus vidas, la madurez alcanzada y constituye el evento nuclear de una nueva subjetividad, según Serrano et al. (43). Ser padre adolescente está estrechamente ligado con la emoción y con sentimientos positivos como alegría, amor, cariño y seguridad, entendiendo que en este acontecer también crecen y maduran (35).

No solamente es un acontecimiento que, para algunos, los certifica como hombres, sino que le proporciona reconocimiento frente a una comunidad que lo acredita como tal. Existe un grupo de padres adolescentes para los cuales el tener un hijo(a) es motivante y se debe vincular al mercado laboral convirtiéndose en el proveedor económico que conllevará a sacarlos adelante y progresar $(35,43,51)$.

La percepción de estos padres en el ejercicio de la crianza puede ser una fuente de identidad masculina, pero resulta fundamental en su construcción la participación del individuo en una actividad generadora de ingresos que se muestre en lo público, espacio en el que él mismo se reconoce como exitoso $\mathrm{y}$, en última instancia, como dominador. Se apoya en dos aspectos: autoridad y capacidad de proveer (52). Por esta razón, algunos adolescentes manifiestan tener temor de no poder asumir el papel de padre, situación que acarrea consecuencias para la propia imagen masculina.

Por lo anterior, pueden llegar a una crisis de identidad, abandonar a su compañera o tornarse violentos, 
presentando sentimientos de angustia, pues al no tener experiencia y frente a la responsabilidad de tener un bebé y no poder proveer, el hombre vive una fase de enfrentamiento que puede afectar su masculinidad frente a otros y frente a sí mismo (50).

Sin embargo, no en todos los padres la percepción es la misma, pues existe un grupo de ellos que, mientras la madre adolescente se configura como cuidadora de su hijo (a) y de su compañero, asumiendo el ejercicio de la crianza de manera obligatoria, para el hombre es sólo una opción que puede tomar o rechazar $(35,44)$. En ocasiones, la aceptación de sí como padre es un proceso lento y difícil para él, que puede lograrse con la comunicación y el apoyo dado por su familia (53).

Por otro lado, existe un grupo de hombres que son invisibilizados durante el ejercicio de la crianza y, aunque el padre adolescente se apropie de nuevas responsabilidades en esta transición de su vida, percibirá estar sumido en sombras y estigmatizado, según Arzola y Lombardo (48), como un padre empequeñecido por abuelos, por suegros y por generaciones de mayor jerarquía en la familia consideradas más capaces que él.

De esta manera, no se les integra en este proceso por entender esta práctica como exclusiva de la mujer y, por lo tanto, no se perciben como padres que ejercen crianza, según lo refieren Meincke y Carraro (54), pues esta percepción es un proceso en constante construcción que se da a través de interacciones que a ellos no se les permite.

Estas percepciones dan cuenta de las singularidades que existen en cada uno de los padres y madres adolescentes, pues mientras para unos el ejercer la crianza durante este curso de vida los hace sentir gratificados(as) y felices, poseer sentimientos de logro por el reconocimiento público y por el estatus que obtienen dentro de su grupo de pares y dentro de la comunidad, sentir haberse convertido en personas maduras, responsables o en hombres o mujeres simbólicamente adultos que han logrado la realización de su vida, para otros son experiencias que infunden sentimientos de miedo y percepciones de incapacidad $\mathrm{y}$ angustia por el desconocimiento y vulnerabilidad social que les rodea.

Una situación relevante en la revisión efectuada es que la percepción de padre adolescente que ejerce la crianza ha ido cambiando con el transcurso del tiempo. Cruzat y Aracena (55), en Chile, refieren que estos padres están experimentando una transición desde el rol tradicional y hegemónico de padre proveedor hacia un padre con necesidades de acercamiento más afectivo al hijo/a y, aunque el eje central y una de las actividades principales que se demarcan es ser el proveedor económico y representar la autoridad, en la mayoría de los casos necesitan compartir e interactuar con sus hijos.

De acuerdo con Celedón y Garri (56), ser padre o madre adolescente puede cruzarse con la vulnerabilidad social en donde se ven interrumpidos los estudios y se debe apresurar la inserción al mundo laboral, afectando "las subjetividades que se despliegan en cada sujeto frente a la irrupción temprana de una función asociada al mundo de los adultos/as". El ser familia adolescente se inicia en la desigualdad frente a los adultos bajo la mirada socio-política en donde el sentirse padre y madre, en donde el construir y sentirse familia sólo por proveer un vínculo afectivo, se muestra insuficiente frente el ojo público (56). Lo anterior puede afectar las percepciones de sí que posea cada padre o madre adolescente en el ejercicio de la crianza.

\section{Interacciones de padres y madres adolescentes en el ejercicio de la crianza}

Como refiere Blúmer (57), "una sociedad humana se compone de personas comprometidas en el acto de vivir. De esta manera, la vida es un proceso de continua actividad en la que los participantes desarrollan líneas de acción ante las innumerables situaciones que han de afrontar". En ese sentido, "el ser humano orienta sus actos hacia las cosas en función de lo que éstas significan para él y el significado de estas cosas se deriva de o surge como consecuencia de la interacción social que cada cual mantiene con el prójimo” (57).

En el caso de padres y madres adolescente en ejercicio de la crianza, cabe resaltar que el desarrollo de esta labor les permite ir aprendiendo y construyéndose como padres o madres, y, en la medida que interactúan con su hijo o hija y con el contexto en general, responder de acuerdo con los significados que para él o ella posea cada persona u objeto.

No obstante, es importante tener en cuenta que las actividades ejercidas en la crianza, especialmente en padres y madres adolescentes, pueden estar influidas por personas allegadas que aconsejan 
ISSN-PRINT

1794-9831

E-ISSN 2322-7028

Vol. 14 No. 2

Jul - Dic 2017

Cúcuta, Colombia cómo interactuar ante las demandas del otro o que simplemente han estado presentes como un modelo que transita de generación en generación. Ante esto, Blúmer (57) menciona que las actividades de los demás intervienen como factores positivos en la formación de su propio comportamiento; sin embargo, ante los actos ajenos, una persona puede abandonar una intención, reconsiderarla, verificarla, cancelarla, intensificarla o sustituirla.

En ese sentido, la acción humana se forma mediante un proceso de interacción del ser humano consigo mismo y, para poder actuar, el individuo tiene que determinar lo que desea, fijarse una meta u objetivo, planear de antemano una línea de comportamiento, advertir e interpretar las acciones ajenas, asumir su propia situación, verificarse a sí mismo con respecto a esto o aquello, concebir lo que se debe hacer en otros casos y, frecuentemente, estimularse ante condiciones que le generan obstáculos o situaciones desalentadoras (57). Esto puede verse reflejado en los padres y madres que, en algunos casos, a pesar de que retoman de sus madres concepciones y formas para asumir su crianza, a su vez, resignifican y modifican el vínculo y tipo de interacción con sus hijos (38). Algunas interacciones que se destacan en los hallazgos de los trabajos revisados son las siguientes:

\section{Con sus hijos}

González y Estupiñán (39) y Rozo et al. (40) han identificado en sus estudios que algunas madres adolescentes son promotoras del cuidado de sus hijos e interactúan con ellos de acuerdo con sus demandas: los estimulan, amamantan, demuestran sensibilidad y apego cuando están recién nacidos, intentan pasar tiempos de calidad con sus hijos e hijas aún a pesar de realizar otras tareas como continuar sus estudios y llevar a cabo las labores del hogar, pues sienten la responsabilidad que demanda ser ama de casa, sin escatimar los esfuerzos por su hijo o hija, lo cual hace que se conviertan en madres humanas competentes ante su familia y su pareja.

Estas adolescentes madres, a las que se hace referencia en los anteriores estudios, se vinculan con su hijo para establecer una relación positiva a través de manifestaciones verbales y físicas como besos, abrazos, caricias y cargarlos. También expresan que enseñarles, hablarles, darles buen trato, salir de paseo al parque, darles lo que necesitan o darles un dulce son demostraciones de cariño hacia sus hijos que se requieren en esta etapa (38).

Existe, a su vez, una correlación positiva y significativa entre la sensibilidad materna y el apego. Es decir, que las respuestas de la madre ante las necesidades del bebé, efectivamente, influyen en la forma como éste se relaciona con ella y con su estilo de respuestas como madre. De otro lado, se identifica que a partir de las expectativas del padre y madre en relación con el sexo de sus hijos, se configuran también sus interacciones con ellos $(35,58)$.

No obstante, se identifican otros hallazgos que evidencian interacciones de las madres con sus hijos en donde ellas se tornan distantes y parecen tener más dificultades para regular sus propios estados emocionales durante esas interacciones, debido a factores como la depresión y las vivencias sociales difíciles que interfieren en la calidad de la relación entre madres e hijos (59).

Con respecto a los padres, es importante hacer hincapié en que no existe una amplia literatura que indague acerca de las interacciones de estos adolescentes con sus hijos e hijas, pues la mayoría de estudios sobre el tema se han enfocado hacia la mujer.

Se identifica que las relaciones de los hombres con sus hijos son esporádicas o se concentran alrededor de la provisión económica, razón por la cual aparece la mujer como la más importante en la vida del niño o niña, considerada como fuente de cariño y de sacrificio (23). Los padres adolescentes se ven, dentro de las interacciones realizadas, más involucrados en actividades relacionadas con los juegos y la recreación (59).

El padre es aquel que protege, provee, forma, educa y representa a la autoridad. "Por otro lado, la paternidad es un lugar donde se construye y se reproduce la masculinidad y donde, estructuralmente, los hombres se colocan en una situación de poder y control sobre los hijos y la mujer" (55).

\section{Con su familia}

El apoyo de la familia, para las madres, es calificado como un elemento facilitador que las hace fuertes y les ayuda a dar respuesta a los cuidados que demandan los hijos(as) (41), además de disminuir sus niveles de estrés ante esta labor (39). Se cita como 
un requerimiento que se hace necesario, además, para continuar sus estudios y, para los padres, este apoyo propicia actitudes favorables y obtención de logros personales y familiares $(49,51)$.

Algunas tareas como la higiene de los niños y niñas, especialmente en su época de recién nacidos, es asignada, en ocasiones, a la madre de la adolescente y es casi normativo que las prácticas de crianza se van generando de manera tradicional, de generación en generación, para realizar estas acciones $(38,39$, $60)$.

A partir de estas delegaciones a la abuela materna, se va configurando otro fenómeno, el abuelazgo, el cual pone en consideración un "modelo social y afectivo" asociado con cuidado, protección, educación, dedicación y manifestaciones de afecto y amor, de manera bidireccional entre abuelos y nietos (16). Además, instala en la cotidianidad familiar la referencia de los consejos por parte de los abuelos y abuelas sobre los aspectos relacionados con la crianza, según Marín y Palacio (61).

El apoyo de las abuelas, identificado por ÁlvarezÁlvarez et al. (62), es tal, que son ellas las que, en muchas ocasiones, toman las decisiones referentes al nieto o nieta, quedando la opinión de la hija en segundo lugar. En este sentido, hay madres que son conscientes de su responsabilidad pero hacen lo que se espera de ellas y confían en las decisiones de su entorno. El apoyo de las madres de las adolescentes en todos los ámbitos se configura como un elemento esencial para una adecuada adaptación a su nuevo papel de madre y para optimizar las oportunidades que le puede otorgar, en determinados contextos de vida, aunque resulte en detrimento de la salud de la abuela (62).

El papel que juegan para la mujer, la familia de origen y la extensa en la asunción de la crianza es relevante, pues estos actores se constituyen en los referentes psicosociales que las mujeres retoman y resignifican como madres. Si bien hay elementos que pueden influir negativamente o limitar la relación madrehijo frente a la crianza y construcción de vínculos en el caso de las madres adolescentes, no es la edad la que determina de forma estructural estas dificultades, sino la ausencia o rompimiento de las redes de apoyo familiar con las que las adolescentes cuentan (38).
En general, la familia se identifica, dentro de los estudios revisados, como una parte del microsistema que tiene papel clave para los padres y madres adolescentes en el ejercicio de la crianza, pero especialmente para el padre, en algunos casos, representa los lazos de apoyo para desarrollar sus capacidades en esta labor $(51,54)$. Este proceso ocurre a partir del conocimiento empírico de las familias, en el que los padres procuran vivir y ejercer la crianza adaptándose a la nueva situación con el apoyo familiar, situación que les permite hacer frente a los retos que les genera el ser padre y continuar con sus proyectos planteados (49).

\section{Interacciones con el contexto laboral, salud, educación, recreación}

En diferentes países del mundo se estima que los padres y madres adolescentes tienen un gran riesgo social, pues no cuentan con los recursos económicos necesarios para atender un grupo familiar y realizar interacciones con el medio en forma adecuada $(58,63)$. Una de las causas de este problema se relaciona con el bajo nivel de escolaridad que poseen, lo cual conlleva a que se empleen en labores que no tienen remuneración suficiente para cubrir sus gastos $(58,64)$.

En Colombia, por ejemplo, se halló que, además de tener menos oportunidades laborales, los padres y madres adolescentes sufren el impacto de la disminución de los ingresos por hora respecto a la población general, en mayor magnitud las mujeres $(12,7 \%)$ en relación con los hombres (5,3\%) (65), datos que representan una realidad a la que se enfrentan estos padres y madres cuando intentan cuidar de su hijo o hija y mantener económicamente los gastos de una nueva familia.

Según Cabello y Reyes (46), "de esta forma, se incorporan como adultos en una sociedad donde el Estado, por un lado, invisibiliza la actividad sexual y condena el embarazo adolescente y, por el otro, paradójicamente, coarta los derechos sexuales y reproductivos mientras coacciona el otorgamiento de alternativas y oportunidades que permitan a los adolescentes un proyecto de vida más allá del embarazo y la unión".

En cifras del Departamento Administrativo Nacional de Estadística (DANE), presentadas en el resumen ejecutivo del Mercado Laboral de la Juventud para
E-ISSN 2322-7028

Vol. 14 No. 2

Jul - Dic 2017

Cúcuta, Colombia 
ISSN-PRINT

1794-9831

E-ISSN 2322-7028

Vol. 14 No. 2

Jul - Dic 2017

Cúcuta, Colombia el trimestre móvil junio-agosto de 2016, puede identificarse que la población joven (14-28 años) tuvo una tasa de ocupación del $48,6 \%$ y una tasa de desempleo general de $15,9 \%$, siendo la de las mujeres de 20,5\% y la de los hombres de $12,5 \%$ (66). En este sentido, son pocas las oportunidades que tienen estas personas en relación con un empleo y, sobre todo, uno de calidad.

En estos datos puede evidenciarse la persistencia, todavía, de la influencia del modelo de marido proveedor y de mujer guardiana de la moral perfilado en el siglo XIX (67), pues se evidencia mayor desempleo para la mujer y tal vez más oportunidades para los hombres, quienes son reconocidos como aquellos que deben proveer económicamente para sus hijos e hijas, pudiendo abandonar incluso sus proyectos escolares y emplearse en mano de obra mal remunerada (65).

Sin embargo, se encuentra que algunas mujeres trabajan, estudian y cumplen labores de crianza, sobre todo cuando el padre no está presente $(38,40)$. Trabajan para garantizar su propio sustento y para el de su hijo e hija sin tener que depender de otra persona (41). Con respecto a esto último, Cruzat y Aracena (55) afirman que "con la incorporación de la mujer al mundo laboral, se han ido reformulando las funciones y labores de madre y, por ende, también las del padre. Es por eso que surge el interrogante de si la necesidad de incorporación a los diferentes procesos de crianza del hijo/a de parte de los varones, responde a una necesidad personal, o bien, han ajustado el discurso petitorio de las mujeres para poder adaptarse a los cambios".

También se encuentra que los padres, a pesar de cumplir funciones de proveedores, continúan sus estudios y trabajan a tiempo parcial (47). Ellos consideran, además, la educación formal como un medio para mejorar la vida y proporcionar un soporte más adecuado para el desarrollo de su familia, como lo establecen Bordignon et al. (68). También Akiko (45) afirma que los adolescentes urbanos requieren más ayuda familiar para el cuidado de sus hijos que los rurales.

Marín y Palacio (61) destacan el papel de las abuelas frente a las necesidades de las madres solteras, pues gracias al apoyo brindado, estas adolescentes logran seguir en el sistema educativo o cumplen el papel de proveedoras económicas. En definitiva, la complejidad del momento actual se expresa en múltiples conflictos, logros precarios, modelos emergentes y paradojas contextuales.

En relación con los espacios de recreación que utilizaba antes de convertirse en padre o madre adolescente, la mayoría de mujeres refieren que se distanciaron de sus amigos y dejaron la vida en la calle para brindar más atención a sus hijos, cambios evaluados de forma positiva por las adolescentes (45). Para algunos hombres, la experiencia de ser padre se asocia con un cambio personal y social (47). Los adolescentes enfrentan ciertos estados que implican cambios en las actividades realizadas en el tiempo libre (por la necesidad de trabajar y obtener ingresos), la necesidad de solicitar ayuda económica a los padres o suegros, fenómeno que conlleva a un posible deterioro en la relación de pareja y obstaculización de sus metas (69).

Dentro de las interacciones con el sistema de salud de padres y madres adolescentes en el ejercicio de la crianza, se observa que se hace alusión a la mujer como usuaria de estos o como la cuidadora que lleva a sus hijos e hijas a controles y que cuida a sus recién nacidos en las salas de cuidado intensivo. Es muy poca la información que existe de padres realizando estas labores $(44,70)$.

Se evidencia, adicionalmente, una invisibilización del padre en el ejercicio de la crianza, pues los estudios centralizan su eje de atención en la madre adolescente y su hijo, pero no se ocupan de lo que vivencia y siente el padre $(40,69)$. Estas familias, para Celedón y Garri (56), son como "un no ser en la agenda pública, lo que conlleva a otro grupo de vulneraciones en términos de no tener y no acceder". Barreto (69) afirma que se hace necesario tener en cuenta las voces de los propios adolescentes varones acerca de la paternidad en la adolescencia, pues esto contribuye en la construcción de políticas públicas orientadas a mostrar al Estado sus dificultades y necesidades y, en contrapartida, la deficiencia que pueden tener las políticas públicas ya existentes.

\section{Desarrollo de políticas públicas en torno a padres y madres adolescentes}

La irrupción como padre o madre en la adolescencia genera un quiebre desde la perspectiva de la movilidad 
social. No tener en cuenta esta situación y sus contextos variados puede conllevar a mantener el estigma y las condiciones de vulnerabilidad que se observan en algunos espacios. En ese sentido, el desafío "es reconocer e incluir las distintas representaciones de ser familia, hoy caracterizadas por su inestabilidad y su capacidad de mutar" (56), lo cual implica "nuevas aperturas a los emergentes sociales, cuidando de no excluir el lugar protagónico de las madres y padres adolescentes en relación al cuidado de sus hijos/as y de sus propias trayectorias" (56).

Con respecto a las acciones que el estado colombiano ha realizado en torno a esta temática, se identifican algunas estrategias y políticas. Según el informe dado por la Dirección del Sistema Nacional de Juventud, Colombia Joven y el Fondo de Población de las Naciones Unidas (UNFPA) (71), se han realizado inversiones por un total de $\$ 21,9$ billones de pesos en adolescentes y jóvenes, cifra que representa el 2,9 \% del producto interno bruto del país.

Igualmente, un $93,5 \%$ de los recursos del gasto específico se invierten exclusivamente en educación (72). Por otra parte, Colombia destina $\$ 9,45$ billones de pesos en gasto ampliado para adolescencia y juventud. Mientras en los territorios, el 71,7 \% de los recursos va a la afiliación en salud, a nivel nacional el $78,5 \%$ se invierte en la atención a la población víctima del conflicto armado (73).

De otra parte, existen normativas que arropan a la población adolescente y que son base de los programas y políticas aprobadas en el país, entre ellas la Ley 1098 de 2006 (Código de Infancia y Adolescencia) que establece como adolescentes a las personas entre los 12 y 18 años (8).

También se encuentra la Ley Estatutaria de Juventud, Ley $N^{\circ} 1622$ de 2013 (9), la cual reconoce, en su artículo 5, como joven a aquella persona "en proceso de consolidación de su autonomía intelectual, física, moral, económica, social y cultural que hace parte de una comunidad política y, en ese sentido, ejerce su ciudadanía", pero, además, estima en el ítem 3 de este mismo artículo que "las realidades y experiencias juveniles son plurales, diversas y heterogéneas" por lo cual los jóvenes deben ser comprendidos "como una construcción cuya subjetividad está siendo transformada por las dinámicas sociales, económicas y políticas de las sociedades y a cuyas sociedades también aportan”.
Esta última norma, Ley 1622 de 2013 (9), utiliza lenguajes que develan cambios que se van dando a nivel normativo, teniendo en cuenta las subjetividades y diversidades que aparecen en los contextos, entendiendo que debe aportarse a la población desde la misma dinámica que se va gestando a nivel social y cultural. Sin embargo, deben notarse los aportes que se han dado para los padres y madres adolescentes o simplemente para los adolescentes, desde el Estado.

Actualmente, existen normativas que hacen referencia al mejoramiento de oportunidades económicas para la población juvenil, dentro de la cual se incluye al grupo etario considerado adolescente. Dentro de esas normas se encuentra el Conpes 173 de 2014 (73) que propone lineamientos generales para propiciar la adecuada inserción laboral, la Ley 1014 de 2006 (74) que establece la red de emprendimiento, la Ley 1429 de 2010 (75) para la formalización y generación de primer empleo y la Ley 1780 de 2016 (76) por medio de la cual se promueve el empleo y el emprendimiento juvenil.

En relación con la salud, se han establecido diferentes normativas y estrategias que intentan dar cobertura a este grupo poblacional, focalizadas en dos temas centrales que se tratan de manera especial: la sexualidad y la prevención del embarazo en adolescentes, los cuales se desarrollan con énfasis en la mujer.

A partir de estos ámbitos, se elaboraron políticas, programas y lineamientos, dentro de los cuales están, por ejemplo, la política en salud sexual y reproductiva (77), los lineamientos como el Conpes Social 147 de 2012 (26) que estableció estrategias para la disminución de embarazo en la adolescencia, los programas como Servicios de Salud Amigables para Adolescentes y Jóvenes (78) que delimita la ruta de atención en asesoría y atención en salud sexual y reproductiva, y el Programa de Control del Joven entre 10 y 29 años (79).

Puede verse que la orientación de estos programas y políticas se ha dado en torno a unas necesidades que se han presentado desde hace varias décadas y, en algunas ocasiones, han sido vistas solamente desde la óptica del estado, considerándose, por ejemplo el embarazo en adolescentes, como un problema de salud pública que se debe combatir, sin diferencias de raza, etnias, edades, culturas, etc. 
ISSN-PRINT

1794-9831

E-ISSN 2322-7028

Vol. 14 No. 2

Jul - Dic 2017

Cúcuta, Colombia
No obstante, es necesario reconocer que, a pesar de todos los esfuerzos, las cifras demuestran que el número anual promedio de hijos de mujeres entre 15 a 19 años, durante el periodo 2003 a 2013, ha sido de 154.707; y el de mujeres de 10 a 14 años sigue aumentando en 6.292 (80). Los datos anteriores demuestran la necesidad de buscar estrategias que apoyen a estas nuevas familias que se encuentran ya en el ejercicio de la crianza (81).

El $27 \%$ de la población colombiana se encuentra entre los 14 y 28 años (57) y de estos, 5.179.937 aproximadamente están entre 12 y los 17 años (82); sin embargo, las situaciones de desigualdad y precariedad son más fuertes en este grupo poblacional.

Estos jóvenes aún no cuentan con suficientes oportunidades. Lo anterior es corroborado por el Conpes 173 de 2014 (73), el cual afirma que "la proporción de jóvenes (porción que incluye la adolescencia) que sólo estudia disminuye drásticamente al aumentar la edad. De $65.4 \%$ (2.3 millones de personas) en el grupo de 14-17 años, baja a $19.3 \%$ en el grupo de 18-21 años (...) (73). Otro aspecto a destacar es la fallida salida de los jóvenes al mercado labora, 1 como lo presenta el sistema territorial de Juventud en Colombia (83)

Este documento indica, además, que "la situación es especialmente crítica para el grupo de 18-21 años (...) En 2011, el 32,8 \% de los jóvenes en condición de pobreza estaba asistiendo a algún programa de formación superior. En contraste, el 87,1\% de los jóvenes de clase alta estaba cursando dicho nivel". (73)

\section{Conclusiones}

Esta revisión demarca la historia que ha tenido la situación de los padres y madres adolescentes, evidenciando que sus percepciones varían de acuerdo con el contexto, en forma particular. No es la edad lo que los hace ser calificados como vulnerables, sino las condiciones y oportunidades que se encuentran a su alrededor.

Sus interacciones con el contexto, igualmente, son resultado de su percepción como adolescente padre o madre. Influye de manera especial su familia, tanto para el reconocimiento de su papel en esta nueva labor como para el logro de sus nuevas tareas y la continuidad de aquellas que desempeñaba antes de ejercer la crianza.

No es que los adolescentes con hijos no deseen continuar sus estudios o que el convertirse en padres y madres en este curso de vida sea motivo para aumentar los círculos de pobreza en las regiones, sino que las condiciones y redes de apoyo con que cuentan son escasas y no le proveen los medios necesarios para asumir las nuevas tareas de padres y madres y continuar con las actividades que le imponen los modelos y sistemas sociales, es decir, existen como padres y madres en un medio que los invisibiliza y estigmatiza.

Para el ejercicio de la crianza es relevante que las y los adolescentes encuentren apoyo en políticas públicas y programas específicos, creados con base en la percepción de esta población, sus interacciones, necesidades, dudas y expectativas.

Lo anterior se puede lograr incluyéndolos en el diseño, ejecución y evaluación de las acciones políticas y de los programas desarrollados o proyectados para atender las situaciones que enfrentan en este periodo del curso de vida.

En ese sentido, puede referirse que la edad no es la única responsable de los problemas de salud, sociales o políticos de un país, sino que son las oportunidades y redes de apoyo con las que cuentan las personas, las que le ayudan a seguir su curso de vida en condiciones dignas y de calidad. En el caso de hombres y mujeres adolescentes que se encuentran en su "paternar" o "maternar" es necesario crear estrategias de apoyo que permitan avanzar en el logro de sus metas propuestas y en las de sus hijos.

La acepción homogeneizante de incapacidad para la crianza en esta población debe analizarse reconociendo singularidades personales y contextuales y examinando, a su vez, las posibilidades que ostentan a su alrededor.

\section{Conflicto de intereses}

Los autores declaran no tener ningún conflicto de intereses. 


\section{Referencias bibliográficas}

1. Borrás-Santisteban T. Adolescencia: definición, vulnerabilidad y oportunidad. CCM [Internet]. 2014 [consultado 23 de Noviembre de 2016]; 18(1): 05-07. Disponible en: http://scielo.sld.cu/scielo. php?script $=$ sci arttext\&pid $=$ S1560-43812014000100002\&lng=es

2. Serrano JM, Pons-Parra RM. El constructivismo hoy: enfoques constructivistas en educación. Revista Electrónica de Investigación Educativa [Internet]. 2011 [Consultado el 8 de agosto de 2016]; 13(1): 1-27. Disponible en: http://redie.uabc.mx/vol13nol/contenido-serranopons.html

3. Duarte-Quapper C. Sociedades adultocéntricas: sobre sus orígenes y reproducción. Última década [Internet]. 2012 [Consultado el 24 de Octubre de 2016]; 20(36): 99-125. Disponible en: http://www. scielo.cl/scielo.php?script=sci_arttext\&pid $=$ S0718-22362012000100005\&lng=es\&nrm=iso

4. Varela-Petito C, Lara C. Jóvenes de hoy, adolescentes de ayer en Uruguay: maternidad y desempeños. Sociedad y Economía [Internet]. 2015 [Consultado el 15 agosto 2016]; (29): 15-37. Disponible en: http://sociedadyeconomia.univalle.edu.co/index.php/sye/article/view/3373

5. Climent GI. Voces, silencios y gritos: Los significados del embarazo en la adolescencia y los estilos parentales educativos. Rev. argent. sociol. [Internet]. 2009 [consultado 12 de septiembre de 2016]; 7(13): 186-213. Disponible en: http://www.scielo.org.ar/scielo.php?script=sci arttext\&pid=S1669$\underline{32482009000200004 \& \operatorname{lng}=\mathrm{es} \& \operatorname{lng}=\mathrm{es}}$

6. Higuita-Gutiérrez LF, Cardona-Arias JA. Concepto de calidad de vida en la adolescencia: una revisión crítica de la literatura. CES Psicol. [Internet]. 2015 [consultado 15 de julio de 2016]; 8(1):155-158. Disponible en: http://www.scielo.org.co/scielo.php?script=sci_arttext\&pid=S201130802015000100011\&lng=en\&nrm=iso

7. Ramos CA. Los paradigmas de la investigación científica. Av.psicol. 2015; 23(1): 1-17.

8. República de Colombia. Congreso de Colombia. Ley 1098 de 2006, noviembre 8, por la cual se expide el Código de la Infancia y la Adolescencia. Diario Oficial No. 46446. Bogotá; 2006.

9. República de Colombia. Congreso de Colombia. Ley 1622 de 2013, abril 29, por medio de la cual se expide el estatuto de ciudadanía juvenil y se dictan otras disposiciones. Diario Oficial No. 48776. Bogotá; 2013.

10. Fondo de las Naciones Unidas para la Infancia (UNICEF). Estado mundial de la Infancia 2011. La adolescencia una época de oportunidades. [Internet]. Nueva York, NY: UNICEF; 2011. [consultado 12 de septiembre de 2016]; p. 134. Disponible en: http://www.unicef.org/honduras/Estado_mundial infancia_2011.pdf

11. Berger PL, Luckman T. La construcción social de la realidad. Argentina: Amorrortu Editores S.A.: 2001.

12. Organización Iberoamericana de Juventud. Convención Iberoamericana de Derechos de los Jóvenes. Acta final. [Internet]. 2005. [consultado 12 de septiembre de 2016]; Disponible en: http://www.oij. org/file upload/publicationsItems/document/20120607115106_98.pdf

13. República de Colombia. Congreso de Colombia. Ley 57 de 1887, mayo 26, por el cual se adopta el Código civil colombiano.

14. Bermúdez DM. Padres Adolescentes y Jóvenes Debates y tensiones. Revista Katálysis, 2016; 19(1), 91-99.

15. Donoso, E., Carvajal, J. A., Vera, C., \& Poblete, J. A. (2014). La edad de la mujer como factor de riesgo de mortalidad materna, fetal, neonatal e infantil. Revista médica de Chile, 142(2), 168-174.

16. Rengifo, A. L. M., \& Palacio, M. C. (2016). La crianza y el cuidado en primera infancia: un escenario familiar de inclusión de los abuelos y las abuelas. Trabajo Social, (18), 159.

17. Martinez, Á. C. (2010). Pautas de crianza y desarrollo socioafectivo en la infancia. Diversitas, 6(1), 111-121.

18. Campo A, Alonso RM, Amador R, Díaz R, Durán MI, Ballesté, I. Maltrato infantil en hijos de madres adolescentes en el Hospital Docente Ginecoobstétrico de Guanabacoa (2005-2006). Rev Cubana Med Gen Integr [Internet]. 2011. [consultado 19 de octubre de 2016]; 27(4): 477-485. Disponible en: http:// 
ISSN-PRINT

$1794-9831$

E-ISSN 2322-7028

Vol. 14 No. 2

Jul - Dic 2017

Cúcuta, Colombia scielo.sld.cu/scielo.php?script=sci arttext\&pid=S0864-21252011000400006\&lng=es\&tlng=es

19. Olavarría Aranguren J y Molina Gutiérrez R. Embarazos en adolescentes, vulnerabilidades y políticas públicas. Polis [Internet]. 2012. [consultado 30 de septiembre de 2016]; (30):1-23. Disponible en: http://polis.revues.org/3802

20. Dáguer C, Riccardi M. Al derecho y al revés. La revolución de los derechos sexuales y reproductivos en Colombia. Bogotá: Printex Impresores; 2005.

21. Red de Prevención del Embarazo Adolescente en Medellín, Proyecto Sol y Luna. Cambios en la vida de los mujeres adolescentes a consecuencia de los embarazos y partos de sus hijas/os nacidos entre los años 2002 y 2004 en Medellín. Medellín: Alcaldía de Medellín, Secretaria de Salud: 2007.

22. Ariza VA. Atar a la sociedad: Adolescencia, riesgo y población en la primera mitad del siglo XX. [Tesis magister]. Colombia: Universidad Nacional de Colombia; 2012.

23. Bernal E, Calero ML. El discurso higiénico como argumento moralizante de la mujer: "La Higiene del Bello Sexo" de Ramón Hernández Poggio (1847). Asclepio [Internet]. 2013 [consultado 21 de noviembre de 2016]; 65(1): 1-15. Disponible en: http://asclepio.revistas.csic.es/index.php/asclepio/ article/view/544/563

24. Zicavo-M N, Fuentealba-V A. Resignificando la paternidad, crianza y masculinidad en padres post divorcio. Revista de Investigación en Psicología, [Internet] 2012 [Consultado 24 de Noviembre de 2016] 15(2): 115-127. Disponible en: http://revistasinvestigacion.unmsm.edu.pe/index.php/psico/ article/view/3693.

25. Vega-Aguirre DC. Análisis de actores claves asociados al desarrollo de políticas públicas para madres adolescentes en Chile. Rev. Gerenc. Polit. Salud, Bogotá (Colombia), 12 (24): 54-72, enero-junio de 2013

26. Consejo Nacional de Política Económica y Social (CONPES), República de Colombia, Departamento de Planeación Nacional. Documento Conpes Social 147 de 2012: "Lineamentos para el desarrollo de una estrategia para la prevención del embarazo en la adolescencia y la promoción de Proyectos de vida para los niños, niñas y jóvenes en edades entre 6 y 19 años”. Bogotá D.C.: CONPES; DNP; 2012.

27. Althabe F, Moore JL, Gibbons L, Berrueta M, Goudar S, Chomba E, et al. Adverse maternal and perinatal outcomes in adolescent pregnancies: The Global Network's Maternal Newborn Health Registry study. Reproductive Health [Internet]. 2015. [consultado 21 de noviembre de 2016]; 12(Suppl 2):S8. Disponible en: http://www.reproductive-health-journal.com/content/12/S2/S8

28. La Rosa AF. Complicaciones en recién nacidos de madres adolescentes tempranas en el Hospital Nacional Arzobispo Loayza de mayo del 2008 a mayo del 2012. Horiz. Med. [Internet]. 2015 [consultado el 24 de noviembre de 2016]; 15(1). Disponible en http://www.scielo.org.pe/scielo. php?script=sci_arttext\&pid=S1727-558X2015000100003\&lng=es\&nrm=iso

29. Gallardo-Lizarazo HR, Guerrero-Romero IS, Niño-González JE, Villaveces-Franco M. Maternity in adolescents and its association with caesarean labor. Rev. cienc. cuidad. 2015; 12(2):11-25

30. Herrera JA, Ersheng G, Shahabuddin AK, Lixia D, Wei Y, Faisal M, et al. Evaluación periódica del riesgo biopsicosocial prenatal en la predicción de las complicaciones maternas y perinatales en Asia 2002-2003. Colombia Médica 2006; 37(2 Suppl 1): 6-14.

31. Miller D, Arvizu V. Ser madre y estudiante. una exploración de las características de las universitarias con hijos y breves notas para su estudio. Revista de la Educación Superior 2016; XLV 1 (177): 17-42.

32. Giddens A. Sociología. Madrid: Alianza Editorial S.A.; 2000.

33. Beaver, K. M. \& Belsky, J. (2012). GeneEnvironment Interaction and the Intergenerational Transmission of ParentingTestinge DifferentialSusceptibility Hypothesis. Psychiatric Quarterly, 83:29-40

34. Aguirre-Dávila, E. Prácticas de crianza, temperamento y comportamiento prosocial de estudiantes de educación básica. Revista Latinoamericana de Ciencias Sociales, Niñez y Juventud 2015;13 (1): 223243

35. Ortega AM. Antes, durante y después del embarazo en adolescente: Proyectos de vida y vivencias sobre su sexualidad. [Tesis magister]. Bogotá D.C.: Universidad Nacional de Colombia; 2013.

36. Caro, P. Los padres "de abajo": La paternidad en los temporeros agrícolas. Revista Sociedad y Equidad 
[Internet]. 2011 [consultado 29 de noviembre de 2016]; 2:123-142

37. Miguélez, A. A. Negociaciones de pareja: los trabajos domésticos, la crianza y la construcción de la maternidad y la paternidad. Papeles del CEIC, International Journal on Collective Identity Research 2016;1(152):1-26.

38. Coronado A, Ortiz N. Rol materno y pautas de crianza en nueve madres adolescentes desde una perspectiva generacional, pertenecientes a las UPA de la Fundación Carla Cristina. EN CLAVE SOCIAL 2013; 2(1): 68-83.

39. González AR, Estupiñán MR. Prácticas de crianza de madres adolescentes de la ciudad de Duitama, Colombia. Investigación y Educación en Enfermería [Internet]. 2010 [consultado 11 de octubre de 2016]. 28(3): 396-404. Disponible en: http://www.redalyc.org/articulo.oa?id=105215721010

40. Rozo L, Rodríguez J, Vergara S. Prácticas de crianza en madres jóvenes con hijos menores de cinco años, estudiantes de una carrera de enfermería de una universidad privada de Bogotá D.C. [Tesis doctoral]. Bogotá D.C.: Pontificia Universidad Javeriana; 2010.

41. Andrade PR, Ribeiro CA, Ohara CVS. Maternidade na adolescência: sonho realizado e expectativas quanto ao futuro. Rev Gaúcha Enferm. [Internet]. 2009[consultado 11 de octubre de 2016]; 30(4): 662-668.

42. Reyes A, Rivera AY, Galicia I. Relación entre el autoconcepto y la percepción de la crianza en madres adolescentes y adultas. Revista Intercontinental de Psicología y Educación 2013; 15(2): 45-73.

43. Serrano J, Sánchez B. Subjetividad materna y paterna adolescente y juvenil. Nómadas (Col) 2000; (13): 265-267.

44. Gómez Á, Gutiérrez ME, Izzedin R, Sánchez LM, Herrera NE, Ballesteros M. Representaciones sociales del embarazo y la maternidad en adolescentes primigestantes y multigestantes en Bogotá. Rev. Salud Pública 2012; 14(2): 189-199.

45. Akiko HL. Maternidad en la Adolescencia en una Comunidad de Bajos Ingresos: Experiencias A Través de Historia Oral. Rev. Latino-Am Enfermagem 2008; 16(2): 280-286.

46. Cabello ML, Reyes DJ. Paternidad adolescente y transición a la adultez: una mirada cualitativa en un contexto de marginación social. Iberóforum. Revista de Ciencias Sociales de la Universidad Iberoamericana. [Internet]. 2011 [consultado 8 de agosto de 2016]; (7)27: 1-27. Disponible en: http:// www.redalyc.org/articulo.oa? id=211019068002

47. Molina R. El padre adolescente, su relación parental y de pareja. Última Década 2011; 19(35): 89-110.

48. Arzola C, Lombardo MJ. Maternidad y paternidad adolescente en Chile. [Tesis magíster]. Chile; Universidad de Chile; 2010.

49. Botero Botero LD, Castrillón Osorio LC. La experiencia de la paternidad en adolescentes. Revista Virtual Universidad Católica del Norte 2015; (46): 89-101.

50. Corrêa ACL, Meincke SMK, Schwartz E, Oliveira AMN, Soares MC, Jardim VMR. Percepções de homens sobre a vivência da paternidade na adolescência: uma perspectiva bioecológica. Rev Gaúcha Enferm. 2016; 37(1): e54692.

51. Izzedin R, Pachajoa A. Pautas, prácticas y creencias acerca de crianza... ayer y hoy. LIBERABIT 2009; 15(2): 109-115.

52. Muñoz Chacon S. En busca del Pater Familias: construcción de identidad masculina y paternidad en adolescentes y jóvenes. En: Burak S. Adolescencia y juventud en América Latina: Editorial Tecnológica de Costa Rica, 2001 pág 243- 265

53. Nunes ME, Meincke MS, Schwartz E, Soares M, Corrêa AC. Paternidade na adolescência: a família como rede social de apoio. Texto - contexto enferm. 2012; 21(2): 313-319.

54. Meincke SM, Carraro TE. Vivência da paternidade na adolescência: sentimentos expressos pela família do pai adolescente. Texto contexto - enferm. [Internet]. 2009 [consultado 11 de octubre de 2016]; 18(1): 83-91. Disponible en: http://www.scielo.br/scielo.php?script=sci_arttext\&pid=S010407072009000100010\&lng

55. Cruzat C, Aracena M. Significado de la paternidad en adolescentes varones del sector sur-oriente de Santiago. Psykhe (Santiago) 2006; 15(1): 29-44. 
ISSN-PRINT

$1794-9831$

E-ISSN 2322-7028

Vol. 14 No. 2

Jul - Dic 2017

Cúcuta, Colombia
56. Celedón R, Garri M. Familias adolescentes: Entre no ser, no tener y no acceder. Psicoperspectivas 2014; 13(2): 67-78.

57. Blumer H. El interaccionismo simbólico: perspectiva y método. Hora Nova S.A. Barcelona, 1981. p. $1-76$.

58. Sena A, Rivera R., Díaz E, Hernández B, Armas N. Caracterización de la lactancia materna en madres adolescentes. Rev Ciencias Médicas 2014; 18(4): 574-589.

59. Komura LA, Silva D. Paternidad y maternidad en la adolescencia, conocimiento científico producido en la última década. av. enferm 2006; 24(2): 13-23.

60. Prieto B, Ruíz CH. Significados durante el puerperio: a partir de prácticas y creencias culturales. Aquichan 2013; 13(1): 7-16.

61. Marín AL, Palacio MC. La crianza y el cuidado en primera infancia: un escenario familiar de inclusión de los abuelos y las abuelas. Trabajo Social [Internet]. 2016 [consultado 11 de octubre de 2016]; 18: 159-176. Disponible en: http://www.revistas.unal.edu.co/index.php/tsocial/article/view/58583

62. Álvarez CÁ, Pastor G, Linares ML, Serrano JS, Rodríguez LR. Maternidad temprana: percepciones e implicaciones de las madres de las adolescentes. Matronas prof. 2014; 15(3): 88-94.

63. Mendoza LA, Arias M, Mendoza LI. Hijo de madre adolescente: riesgos, morbilidad y mortalidad neonatal. Rev. chil. obstet. ginecol. 2012; 77(5): 375-382.

64. Varela C, Fostik A. Maternidad adolescente en el Uruguay:¿ transición anticipada y precaria a la adultez? Revista Latinoamericana de Población 2015; 5(8): 115-140.

65. Gómez C. Consecuencias de ser padre a temprana edad sobre los ingresos: Caso colombiano, Ensayos sobre Política Económica [Internet]. 2016 [consultado 11 de octubre de 2016]; 34: 103-125. Disponible en: http://www.sciencedirect.com/science/article/pii/S0120448316300203 http://dx.doi.org/10.1016/j. espe.2016.02.002.

66. DANE. Resumen ejecutivo. Mercado Laboral de la Juventud (14 a 28 años). Junio-agosto 2015 [Internet]. 2015 [consultado 11 de octubre de 2016]. Disponible en: https:/www.dane.gov.co/files/ investigaciones/boletines/ech/juventud/Bol_eje_juventud_jun_ago15.pdf

67. Agirre, A. Negociaciones de pareja: los trabajos domésticos, la crianza y la construcción de la maternidad y la paternidad. Papeles del CEIC. International Journal on Collective Identity Research, 2016;1(152):1-27

68. Bordignon SS, Meincke SM, Soares MC, Schwartz E, Barlem ELD, Lunardi VL. Paternidade na adolescência no contexto dos serviços de saúde, escola e comunidade. Texto Contexto Enferm. 2014; 23(4): 979-986.

69. Barreto J. La relevancia del conocimiento de las representaciones sociales de los adolescentes varones acerca de la paternidad en la adolescencia para el desarrollo de políticas públicas. Subjetividad y Procesos Cognitivos 2013; 17(2): 17-36.

70. Zunino Carlos, García Loreley, Boulay Marie, Pérez Walter. Programa de protección de madres adolescentes de niños hospitalizados "Cuidando a las cuidadoras": Primeros dos años de experiencia. Arch. Pediatr. Urug. [Internet]. 2016 [consultado 24 de Noviembre de 2016] ; 87( Suppl 1 ): S48-S54. Disponible en: http://www.scielo.edu.uy/scielo.php?script=sci_arttext\&pid=S1688$12492016000500007 \& \operatorname{lng}=\mathrm{es}$.

71. Presidencia de la República de Colombia, Colombia Joven. Zona informativa. De interés juvenil. Colombia invierte el 2,9\% de su PIB en adolescencia y juventud. [Internet]. 2016 [consultado 24 de Noviembre de 2016]. Disponible en: http://www.colombiajoven.gov.co/noticias/2016/ Paginas/160707 informe-juventud.aspx

72. Ministerio de Salud y Protección Social de Colombia, Profamilia, Organización Internacional para las Migraciones (OIM), Fondo de Población de las Naciones Unidas (UNFPA). Política Nacional de Sexualidad, Derechos Sexuales y Derechos Reproductivos. [Internet]. 2015 Disponible en: https:// www.minsalud.gov.co/sites/rid/Lists/BibliotecaDigital/RIDE/DE/LIBRO \%20POLITICA \%20 SEXUAL\%20SEPT\%2010.pdf

73. Consejo Nacional de política Económica y social, Republica de Colombia, Departamento Nacional de 
Planeación. Conpes 173 de 2014)

74. República de Colombia. Congreso de Colombia. Ley 1014 de 2006, enero 26, por la cual se establece el fomento a la cultura del emprendimiento. Diario oficial N 46.164. Bogotá; 2006

75. República de Colombia. Congreso de Colombia. Ley 1429 de 2010, diciembre 29, por la cual se expide la ley de Formalización y Generación de Empleo. Diario Oficial № 47.937. Bogotá; 2010

E-ISSN 2322-7028

Vol. 14 No. 2

Jul - Dic 2017

Cúcuta, Colombia

76. República de Colombia. Congreso de Colombia. Ley 1780 de 2016, mayo 2, por medio de la cual se promueve el empleo y el emprendimiento juvenil, se generan medidas para superar barreras de acceso al mercado de trabajo y se dictan otras disposiciones. Cartagena; 2016

77. República de Colombia, Ministerio de Protección Social. Política Nacional de Salud Sexual y reproductiva, 2003

78. Luengo MX, Millán T, Zepeda A, Tijero M. Adolescentes urbanos: conocimientos sobre la atención de salud sexual y reproductiva. Rev. chil. pediatr. [Internet]. 2012 [consultado 24 de noviembre de 2016];83(6):540-551. Disponible en: http://www.scielo.cl/scielo.php?script=sci_arttext\&pid=S037041062012000600003\&lng=es.

79. Ministerio de Salud. Programa de Detección de alteraciones del desarrollo del joven entre 10 y 29 años.

80. Presidencia de la República de Colombia, Colombia Joven. Instituto Nacional Demócrata para asuntos internacionales (NDI). Sistema territorial de juventud herramientas metodológicas 3. Juventud en los planes de desarrollo territoriales. Orientaciones para la inclusión e incorporación de las temáticas juveniles. [Internet]. Colombia: NDI; 2015 [consultado 24 de Noviembre de 2016]. Disponible en: $\quad$ http://mountainbike.com.co/modulo5/wp-content/uploads/2016/03/160410 juventud-planesdesarrollo-vWEB-2.pdf

81. Rico de Coronel AC, Castillo C, Benítez CM, Medina O. Programa de atención integral a la adolescente gestante. Ciencia y Cuidado. 2013; 10(1):67-77.

82. Fondo de las Naciones Unidas para la Infancia (UNICEF) Colombia. Análisis de la situación de la infancia y la adolescencia en Colombia 2010-2014. [Internet]. 2015 [consultado 24 de Noviembre de 2016] Disponible en: http://www.unicef.org.co/sitan/assets/pdf/sitan.pdf

83. Presidencia de la República de Colombia, Colombia Joven. Instituto Nacional Demócrata para asuntos internacionales (NDI). Sistema territorial de juventud herramientas metodológicas 3. Juventud en los planes de desarrollo territoriales. Orientaciones para la inclusión e incorporación de las temáticas juveniles. [Internet]. Colombia: NDI; 2015 [consultado 24 de Noviembre de 2016] Disponible en: $\quad$ http://mountainbike.com.co/modulo5/wp-content/uploads/2016/03/160410 juventud-planesdesarrollo-vWEB-2.pdf 